\title{
Mortality from non-malignant respiratory diseases among workers in the Norwegian silicon carbide industry: associations with dust exposure
}

\author{
Merete Drevvatne Bugge, ${ }^{1}$ Solveig Føreland, ${ }^{2,3,4}$ Kristina Kjærheim, ${ }^{5}$ Wijnand Eduard, ${ }^{2}$ \\ Jan Ivar Martinsen, ${ }^{5}$ Helge Kjuus ${ }^{1}$
}

${ }^{1}$ Department of Occupational Medicine and Epidemiology, National Institute of

Occupational Health, Oslo, Norway

${ }^{2}$ Department of Chemical and Biological Work Environment, National Institute of

Occupational Health, Oslo, Norway

${ }^{3}$ Department of Occupational Medicine, St. Olav's Hospital, Trondheim University Hospital, Trondheim, Norway

${ }^{4}$ Department of Public Health and General Practice, Faculty of Medicine, Norwegian University of Science and Technology, Trondheim, Norway ${ }^{5}$ Department of Aetiological Research, Institute of Population-based Cancer Research, Cancer Registry of Norway, Oslo, Norway

\section{Correspondence to} Merete Drevvatne Bugge, National Institute of Occupational Health, Pb. 8149 Dep, Oslo N-0033, Norway; mdb@stami.no

Accepted 2 February 2011 Published Online First 1 March 2011

\section{ABSTRACT}

Objectives Increased mortality from asthma, chronic bronchitis and emphysema has previously been reported among workers in the silicon carbide (SiC) industry. The objective of the present study was to evaluate the influence of specific exposure factors on mortality from obstructive lung diseases (OLD), using a newly revised job-exposure matrix.

Materials and methods 1687 long-term workers employed in 1913-2003 in the Norwegian SiC industry were characterised with respect to cumulative exposure to quartz, cristobalite, SiC particles and SiC fibres. Standardised mortality ratios (SMRs) for underlying causes of death, 1951-2007, were calculated stratified by category of cumulative exposure, and Poisson regression analyses of OLD were performed using cumulative exposure variables.

Results An increased total mortality (SMR 1.1, 95\% Cl 1.0 to 1.2) and increased mortality from cancer, non-malignant respiratory diseases and external factors, were observed. The SMR of OLD was increased at the highest level of cumulative exposure to all investigated exposure factors. In the internal analyses, a twofold increased risk of OLD was observed with increasing levels of cumulative exposure to SiC particles. In a multivariate model, SiC particles showed the most stable increased risk estimate when controlled for other exposure factors, among workers with less than 15 years of employment. Among workers with more than 15 years of employment, crystalline silica, primarily cristobalite, seemed to be the most important exposure factor.

Conclusion Exposure to $\mathrm{SiC}$ and crystalline silica may contribute to OLD development among SiC industry workers in different time windows, and possibly through different mechanisms.

\section{INTRODUCTION}

The question of adverse health effects among workers in the silicon carbide ( $\mathrm{SiC}$ ) industry was already being addressed in the 1920s. Winslow et al described an excess of tuberculosis among the workers, and pointed to dust in the working atmosphere as a possible cause. ${ }^{1}$ In 1949, Bruusgaard showed that workers in the $\mathrm{SiC}$ industry had a high prevalence of silicosis, and that many of the workers who had developed silicosis seemed not to have been exposed to crystalline silica but only to $\mathrm{SiC}$

\section{What this paper adds}

- Workers in the silicon carbide industry have increased mortality from non-malignant respiratory diseases.

- The specific causal factors of this increase have not previously been known.

- The present study shows that several exposure factors, including silicon carbide dust and crystalline silica, may contribute to the increased mortality.

- Dust exposure control in the silicon carbide industry is essential.

dust. $^{2}$ Two mortality studies have been performed in this industry. In 1994, Infante-Rivard et al found a significantly increased risk of non-malignant respiratory diseases (standardised mortality ratio (SMR) 2.0, 95\% CI 1.2 to 3.2) among workers in the Canadian $\mathrm{SiC}$ industry. ${ }^{3}$ The risk increased with increasing exposure to total dust. In 2002, Romundstad et al published a mortality study in the Norwegian SiC industry, with similar findings-an increased risk of total mortality (SMR 1.1, 95\% CI 1.1 to 1.2 ) and mortality from non-malignant respiratory diseases (SMR $1.4,95 \%$ CI 1.1 to 1.7$){ }^{4}$ Specifically, the combined risk of asthma, chronic bronchitis and emphysema was elevated, with an SMR of 2.2 (95\% CI 1.6 to 3.0), and an increased risk with increasing level of exposure to total dust was also observed. ${ }^{4}$ However, the exposure data in these two studies were limited.

In the present follow-up of the Norwegian cohort, a revised and extended, retrospective job-exposure matrix (JEM) has been developed, based on more than 8500 samples of total dust and dust fractions (Føreland et al, 2011, submitted). This paper presents an update of the previous Norwegian mortality study, ${ }^{4}$ with an additional 11 years of follow-up and improved exposure estimates from the revised JEM. As the risk of non-malignant pulmonary diseases was increased in both previous mortality studies, ${ }^{34}$ we will address this issue specifically, and study the association of mortality from obstructive lung disease (OLD) with exposure to fibres and crystalline components in the dust. The effect of long-term cumulative exposure is also addressed. 


\section{MATERIALS AND METHODS SiC production}

$\mathrm{SiC}$ is produced by the Acheson method. ${ }^{5}$ A mixture of finely ground quartz sand and petroleum coke, often with additives such as sawdust and aluminium oxide, is placed in open furnaces. The mixture is electrically heated by a graphite core to over $2500^{\circ} \mathrm{C}$ close to the core and about $1000^{\circ} \mathrm{C}$ on the periphery. In the region close to the core, silicon dioxide is in the gas phase and reacts with coke to form $\mathrm{SiC}$ and carbon monoxide. The thermal process lasts about $48 \mathrm{~h}$, and at the end the zone closest to the core consists of a thick layer of loosely knitted SiC crystals, whereas the periphery consists of unreacted material. The zone between those two layers contains partially reacted material, and here fibrous $\mathrm{SiC}$ is found. ${ }^{6}$ The unreacted and partially reacted material is separated from the $\mathrm{SiC}$ fraction, and is reused as raw material. Through several crushing and washing processes the crude $\mathrm{SiC}$ is refined into different grain size fractions, according to desired end use. The production of $\mathrm{SiC}$ is energy intensive, and Norway, with its abundant supply of hydro electric power, got its first $\mathrm{SiC}$ plant in 1913. In the 1960s, two additional plants were established.

\section{Cohort design and follow-up}

The study population was based on a previously established cohort of men employed in 1942 and later, with $\geq 6$ months of total employment in the Norwegian SiC industry between 1913 and 2003, and alive after 1 January 1953, giving 2631 individuals in total. ${ }^{7}$ As data on causes of death were available from 1 January 1951, up to two extra person-years of follow-up were added for all workers employed before 1953 and alive between 1951 and 1953. In addition, six workers who died between 1951 and 1953 were added to the cohort. The information available for this extended cohort of 2637 men included name, date of birth, employment periods with job codes, and smoking status (ever/never-smoker and unknown). For workers alive in 1960 or born later, the Norwegian unique 11-digit personal identification number was also available.

Data on causes of death were available from 1 January 1951. For the period 1960-2007, the cohort was linked, via the 11-digit identification number, with the Norwegian National Population Register and the Norwegian Causes of Death Register to obtain dates of death and emigration, and causes of death. To obtain causes of death before 1960, linkage with the individual's name and date of birth was performed. The Regional Committee for Medical Research Ethics and the Norwegian Data Inspectorate endorsed the study.

Eight workers registered as dead in the Norwegian National Population Register had no death diagnoses in the Norwegian Causes of Death Register, and were thus excluded from the cohort. A recent cancer study in the Norwegian SiC industry found different risk profiles between short- and long-term workers, probably due to a combination of occupational and lifestyle factors. ${ }^{7}$ It was therefore decided to base the present follow-up on workers with a total employment of $\geq 3$ years, giving 1687 men in total.

The diagnoses from the Causes of Death Register were coded in International Classification of Diseases (ICD)-6 through ICD-10, depending on the date of diagnosis. Due to changes in the coding system, and changes in diagnostic trends concerning OLD during the years, ${ }^{8}$ we chose to pool diagnoses related to asthma, chronic bronchitis, emphysema and chronic obstructive pulmonary disease (COPD) into OLD. ${ }^{9}$ In the Norwegian Causes of Death Register, both underlying and contributing causes of death are collected from the death certificate. The underlying cause of death is a disease or injury that initiated the train of events leading to death. A contributory cause of death is a condition that contributes to the fatal outcome, but which is not related to the disease or condition directly causing death.

The follow-up of mortality among the long-term workers started after 3 years of total employment duration, or from 1 January 1951 if the 3 years of employment duration was reached before that date, and lasted until 31 December 2007 or the time of death or emigration. The long-term workers contributed 42689 person-years at risk to the study.

\section{The JEM}

A historical JEM was developed for all job groups in each of the three plants, for all years since the start of production. The development of the JEM was based partly on previous dust measurements, performed both for surveillance by the three plants and in connection with studies performed by the Norwegian National Institute of Occupational Health. Altogether more than 3300 total dust measurements from 1967 to 2005 were available, but few measurements (about 300) of specific agents. Hence, the modelling of historical exposure levels was based on the total dust measurements. To predict exposure to specific dust components, a comprehensive comparative exposure assessment study was performed in connection with the present project during 2002-2003, and included 600-700 samples of total dust collected in parallel with fibres and respirable dust. The respirable dust samples were analysed for quartz, cristobalite and non-fibrous SiC content. This study added almost 5000 measurements to the exposure data. ${ }^{10}$ Mixed effect models were constructed to establish the relationships between total dust and specific components, and these models were used to compute historical job type-specific exposure estimates of the dust components. Information from the plants about technological changes and changes in working hours was used to estimate relative changes in total dust exposure during the earliest years of production, when no measurement data were available. Based on the assumption (with a few exceptions) that the composition of the dust did not change over time, group mean exposures to the specific components could be estimated from total dust, in about 80 job groups, distributed over the three Norwegian $\mathrm{SiC}$ plants, for each year of production in the period 1913-2003. The exposure estimation process is described in detail elsewhere (Føreland et al, 2011, submitted). High correlation between exposure to the different agents in the previous JEM made it difficult to separate potential effects posed by different types of exposures. ${ }^{4}$ In the revised JEM the correlation coefficients were moderate to low except between total dust and respirable dust, providing the potential for better differentiation of any harmful effects from exposure factors.

Through linkage via job code and year between the cohort database and the JEM, we were able to estimate each person's time weighted average exposure to each investigated dust component during each year of employment in the $\mathrm{SiC}$ industry. The individual annual exposure was summed, giving estimates of cumulative exposure to total and respirable dust, respirable quartz, cristobalite and $\mathrm{SiC}$ particles, and $\mathrm{SiC}$ fibres, for each person-year of follow-up.

\section{Departments and job groups}

The main departments of $\mathrm{SiC}$ plants are as follows. (i) The furnace department, where workers are exposed to raw materials (coke and quartz), agents formed during the furnace process (cristobalite (a high temperature crystal phase of silica) and $\mathrm{SiC}$ fibres) and the end product (non-fibrous $\mathrm{SiC}$ ). In addition, other 
exposure factors are likely to be present: organic compounds such as polycyclic aromatic hydrocarbons and graphite, carbon monoxide and sulphur dioxide gases, and in earlier periods, asbestos. (ii) The processing department, where the $\mathrm{SiC}$ is refined into size fractions. Here, non-fibrous $\mathrm{SiC}$ is the main exposure factor, but agents from the partly reacted material contaminate the crude $\mathrm{SiC}$ and are also present. (iii) The maintenance department, which mainly consists of electricians and mechanics, working in both the furnace and processing departments. (iv) Other workers, including low exposed personnel, for example laboratory workers and stockhouse workers. (v) Office workers. As the two latter groups are quite small, we have combined them in the analyses.

For the analyses stratified by department, we chose to allocate department affiliation to those who according to our knowledge had worked in one department only. Workers with employment in more than one department, or with unknown affiliation, were allocated to a 'mixed' group. The years of employment in this mixed group were distributed almost equally in furnace (2810 years) and processing (2675 years) departments, in addition to 2181 years in maintenance and 1305 years in 'other, low exposed' jobs.

Figure 1 shows the mean total dust levels, per 10-year period, experienced by the long-term workers in the present cohort, by department.

The cumulative exposures to total and respirable dust, respirable $\mathrm{SiC}$, quartz and cristobalite, and fibres among workers in the different departments are shown in table 1. Arithmetic means are estimated from geometric means as described by Seixas et al. ${ }^{11}$

\section{Data analysis}

SMR were calculated as the ratio between observed and expected numbers of deaths. Expected numbers were based on national mortality rates, calculated from national incidences of underlying causes of death and population numbers (available for 1951-2006), summed in 5-year age and period groups. For 2007 we used the same national rates as for 2006, as an approximation. Cumulative exposure to all six exposure factors was categorised in three groups (low, medium and high) ensuring equal numbers of person-years of follow-up in each category. We performed SMR analyses of the main diagnostic groups overall, and of OLD stratified by department and by category of cumulative exposure. We calculated 95\% CIs assuming a Poisson distribution of the observed numbers.

The correlations between exposure variables were examined using Pearson's correlation coefficients between the log-transformed cumulative exposures.

In order to study possible exposure-response associations, we performed Poisson regression analyses of OLD related to categories of exposure, adjusted for age (dichotomised at 75 years,

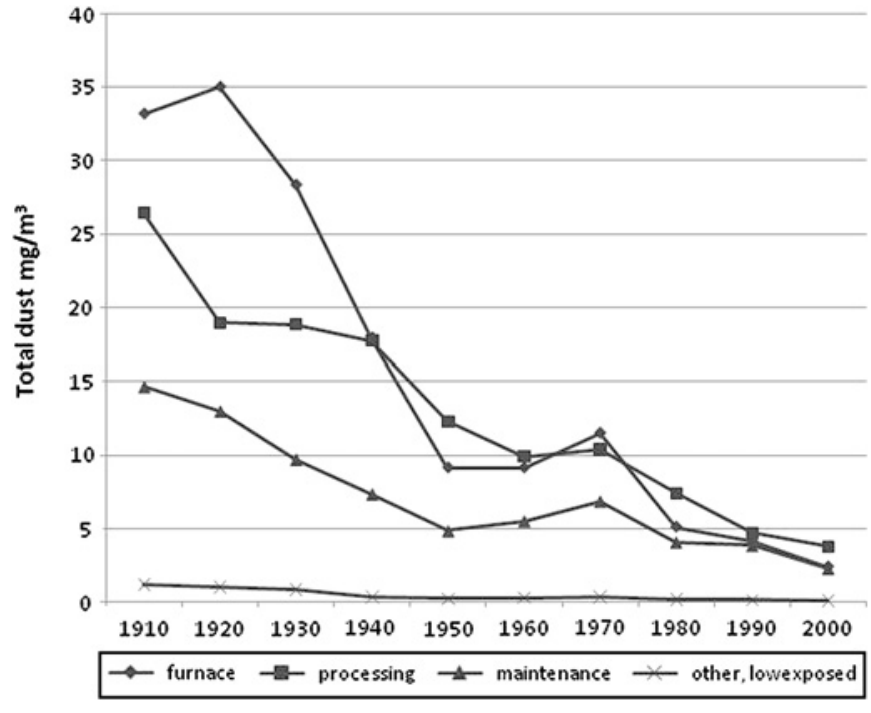

Figure 1 Mean total dust exposure per 10-year period in a cohort of 1687 long-term silicon carbide industry workers, by department.

due to the high age of those dying with OLD), smoking (never, ever and unknown) and period of diagnosis (before/after 1990). In the internal analyses we, as an alternative to OLD as underlying cause of death (OLD-u), used OLD as underlying or contributing cause of death (OLD-uc), to increase the number of cases and thereby the power of the analyses. ${ }^{12}$ Tests of trend were done by fitting a Poisson regression model for the linear effect of the exposure variables, assuming a constant increase in the log rate ratio per exposure category level. ${ }^{13}$ Confounding between the four specific exposure factors (respirable quartz, cristobalite and $\mathrm{SiC}$, and fibres) was studied by modelling Poisson regression analyses with two exposure variables at a time. Continuous cumulative exposure to each exposure factor was log-transformed, due to skewed distribution of the exposure. In order to test the null hypothesis that there was no effect of the second exposure variable after controlling for the first, likelihood ratio testing was performed comparing the model containing one exposure factor with a model containing two exposure factors. These analyses were done among ever-smokers only, stratified by total employment duration $(</ \geq 15$ years) in order to study the effect of long-term cumulative exposure, and adjusted for age and period of diagnosis. In these analyses, follow-up of the group with $\geq 15$ years of employment started after 15 years of total employment.

All statistical analyses were performed using Stata 11.

\section{RESULTS}

Cohort characteristics and the distribution of deaths due to OLD for the 1687 long-term workers are presented in table 2 .

Table 1 Mean cumulative exposure, at end of follow-up, to investigated exposure factors among 1687 male long-term workers employed in 1913-2003 in the Norwegian silicon carbide industry, by department affiliation

\begin{tabular}{|c|c|c|c|c|c|c|c|c|c|c|c|c|c|c|c|c|c|c|}
\hline Department & \multicolumn{3}{|c|}{$\begin{array}{l}\text { Total dust } \\
\left(\mathrm{mg} \times \text { years } / \mathrm{m}^{3}\right)\end{array}$} & \multicolumn{3}{|c|}{$\begin{array}{l}\text { Respirable dust } \\
\left(\mathbf{m g} \times \text { years } / \mathrm{m}^{3}\right)\end{array}$} & \multicolumn{3}{|c|}{$\begin{array}{l}\text { Respirable SiC } \\
\left(\mathbf{m g} \times \text { years } / \mathbf{m}^{3}\right)\end{array}$} & \multicolumn{3}{|c|}{$\begin{array}{l}\text { Respirable quartz } \\
\left(\mathrm{mg} \times \text { years } / \mathrm{m}^{3}\right)\end{array}$} & \multicolumn{3}{|c|}{$\begin{array}{l}\text { Respirable cristobalite } \\
\left(\mathrm{mg} \times \text { years } / \mathrm{m}^{3}\right)\end{array}$} & \multicolumn{3}{|c|}{$\begin{array}{l}\text { Fibre } \\
\left(\text { fibres } \times \text { years } / \mathrm{cm}^{3} \text { ) }\right.\end{array}$} \\
\hline Furnace & 52 & 3.6 & 120 & 7.0 & 3.2 & 14 & 1.0 & 4.7 & 3.3 & 0.058 & 4.1 & 0.16 & 0.10 & 5.9 & 0.48 & 2.5 & 3.2 & 4.9 \\
\hline Maintenance & 56 & 2.5 & 85 & 7.6 & 2.5 & 12 & 1.6 & 2.5 & 2.4 & 0.032 & 2.7 & 0.052 & 0.013 & 3.0 & 0.024 & 1.5 & 3.1 & 2.8 \\
\hline Other, low exposed & 2.0 & 4.6 & 6.4 & 0.28 & 4.0 & 0.73 & 0.028 & 6.7 & 0.17 & 0.0019 & 4.5 & 0.0059 & 0.0026 & 4.6 & 0.0083 & 0.021 & 6.6 & 0.12 \\
\hline Mixed & 85 & 2.9 & 150 & 10 & 2.6 & 16 & 2.9 & 3.5 & 6.4 & 0.054 & 3.4 & 0.11 & 0.056 & 5.6 & 0.25 & 2.0 & 3.5 & 4.4 \\
\hline
\end{tabular}

$\mathrm{AM}$, arithmetic mean estimated from the geometric mean and the geometric $\mathrm{SD}^{11}$; GM, geometric mean; GSD, geometric SD; SiC, silicon carbide. 
Table 2 Cohort characteristics and distribution of obstructive lung diseases as underlying (OLD-u) and underlying or contributing (OLD-uc) cause of death 1951-2007, for 1687 male long-term workers in the Norwegian silicon carbide industry employed 1913-2003

\begin{tabular}{lrrrr}
\hline & N & Person-years & OLD-u & OLD-uc \\
\hline Year of birth & & & & \\
Before 1920 & 513 & 12960 & 24 & 40 \\
$1920-1939$ & 418 & 12932 & 21 & 27 \\
$1940-1959$ & 483 & 12769 & 0 & 0 \\
1960-1980 & 273 & 4028 & 0 & 0 \\
Year of first employment & & & & \\
Before 1940 & 185 & 4668 & 6 & 9 \\
1940-1959 & 295 & 9941 & 14 & 24 \\
1960-1979 & 770 & 21108 & 25 & 34 \\
1980-2000 & 437 & 6972 & 0 & 0 \\
Year of death & & & & \\
1951-1969 & 96 & 1006 & 2 & 3 \\
1970-1989 & 309 & 6507 & 12 & 15 \\
1990-2007 & 383 & 12133 & 31 & 49 \\
Alive & 899 & 23042 & - & - \\
Smoking & & & & \\
Never-smokers & 359 & 9187 & 3 & 8 \\
Ever-smokers & 1162 & 30474 & 38 & 55 \\
Unknown & 166 & 3028 & 4 & 4 \\
Department affiliation & & & & \\
Furnace & 386 & 9226 & 13 & 20 \\
Processing & 346 & 7751 & 9 & 15 \\
Maintenance & 314 & 7978 & 4 & 7 \\
Low exposed & 175 & 5012 & 1 & 1 \\
Mixed & 466 & 12723 & 18 & 24 \\
\hline
\end{tabular}

We observed both an increased total mortality risk (SMR 1.1, $95 \%$ CI 1.0 to $1.2 ; 788$ cases) and increased risks of cancer (SMR $1.2,95 \%$ CI 1.0 to $1.4 ; 201$ cases), respiratory diseases (SMR 1.6, $95 \%$ CI 1.3 to 2.0; 91 cases) and external causes (SMR 1.5, 95\% CI 1.1 to $2.0 ; 44$ cases). In addition, excess risks were found for the respiratory subgroups OLD (SMR 2.0, 95\% CI 1.5 to $2.7 ; 45$ cases), pneumonia (SMR 1.4, 95\% CI 1.0 to $1.9 ; 38$ cases) and pneumoconiosis (SMR 15, 95\% CI 7.0 to 31; 7 cases). For circulatory diseases the SMR was 1.0 (95\% CI 0.9 to $1.2 ; 347$ cases), for digestive diseases 1.1 (95\% CI 0.7 to $1.7 ; 17$ cases) and for other diagnoses 0.9 (95\% CI 0.7 to 1.1 ; 88 cases). (Not shown in table.)

The increased risk of mortality from OLD was linked to employment in the two production departments: furnace (SMR 2.7, 95\% CI 1.6 to 4.6 ; 13 cases) and processing (SMR 2.2, 95\% CI 1.2 to $4.3 ; 9$ cases). There was no increase in mortality from OLD among workers with affiliation to the maintenance (SMR $1.0,95 \%$ CI 0.4 to 2.7 ; 4 cases) or 'other, low-exposed' (SMR 0.4, $95 \%$ CI 0.1 to 2.6 ; 1 case) departments. Workers with employment in more than one department (SMR 2.5, 95\% CI 1.6 to 4.0; 18 cases) had a risk almost as high as furnace workers. (Not shown in table.)

Table 3 presents the analyses of OLD related to categories of cumulative exposure to total dust, respirable dust, respirable $\mathrm{SiC}$, quartz and cristobalite, and fibres. The SMR was significantly increased at the two higher levels of exposure to total dust, cristobalite, $\mathrm{SiC}$ dust and respirable dust, at all levels of exposure to fibres, and at the lowest and highest level of exposure to quartz. Internal analyses in models of ever-smokers using OLD as underlying cause of death showed significantly increased risks at the two higher levels of exposure to $\mathrm{SiC}$ (incidence rate ratio (IRR) 3.1 and 3.4, respectively), with a significant trend $\left(p_{\text {trend }}=0.03\right)$. Using OLD as underlying or contributing cause of death, the IRR estimates for $\mathrm{SiC}$ were somewhat reduced, still with a significant trend $\left(p_{\text {trend }}=0.02\right)$. This last analysis also showed a significant exposure-response trend for total dust $\left(p_{\text {trend }}=0.03\right)$ and cristobalite $\left(p_{\text {trend }}=0.05\right)$. In analyses stratified by total employment duration in the $\mathrm{SiC}$ industry, there was a significant exposure-response trend for $\mathrm{SiC}$ dust among workers with less than 15 years of employment. In contrast, among workers with more than 15 years of employment, there were significant exposure-response trends for total dust and cristobalite, and a borderline significant trend for quartz.

Table 4 shows the results from analyses including two logtransformed cumulative exposure variables in models of eversmokers only. Correlations between cumulative exposure to the investigated specific exposure variables were at a moderate level $(\leq 0.75)$, except the correlations between quartz and fibre exposure (Corr Pearson 0.76-0.83). In the subcohort of eversmokers, $\mathrm{SiC}$ was the exposure factor with the highest risk estimate when studied as an isolated log-transformed exposure factor. This risk estimate remained at the same level when fibres, quartz or cristobalite were included in the model. Similarly, among workers with less than 15 years of employment, the risk estimate was highest for $\mathrm{SiC}$, and was not changed when other exposure factors were included in the model. $\mathrm{SiC}$ was, however, highly correlated to total dust (Corr Pearson $_{\text {0.88-0.93), and by including }}$ total dust in the model, the effect of $\mathrm{SiC}$ was reduced, both among all ever-smokers (IRR 1.1) and among workers with less than 15 years of employment (IRR 1.5; not shown in the table). Among workers with more than 15 years of employment, risk estimates were highest and most stable for the two crystalline silica agents, cristobalite and quartz. When including both cristobalite and quartz in the model, the effect of quartz was reduced, and cristobalite seemed to be the most important factor. The inclusion of total dust did not change these estimates.

These analyses were performed using OLD as underlying or contributing cause of death (OLD-uc). Analyses using OLD as underlying cause of death (OLD-u) gave the same results overall, but with less precision (results not shown).

\section{DISCUSSION}

In a 57-year follow-up of mortality among 1687 long-term workers in the Norwegian SiC industry, we have found increased risks of mortality from all causes, cancer, external factors and non-malignant respiratory diseases, including OLD. SMR analyses of OLD stratified by category of cumulative dust exposure showed significantly increased SMR with higher levels of exposure to all investigated exposure factors. Internal analyses indicated that $\mathrm{SiC}$ was the exposure factor with the highest risk estimate among workers with less than 15 years of employment, and cristobalite seemed to be the most important factor among workers with more than 15 years of employment.

In the previous mortality study in the Norwegian $\mathrm{SiC}$ industry, an excess mortality from asthma, emphysema and chronic bronchitis combined was found, with an exposureresponse association to total dust exposure. High correlations between exposure to the various agents specified in the JEM precluded differentiation between the potential effects of these agents. ${ }^{4}$ The present study confirmed the main results from the previous study, and the revised and extended JEM made it possible to better distinguish between the effects of different exposure factors.

The improved JEM used in this study provided estimates of exposure to specific exposure factors in more than 80 job groups during almost 100 years of industrial activity. Cumulative exposures to total dust, respirable dust and components in the 
Table 3 Standardised mortality ratios (SMR) and Poisson regression analyses (incidence rate ratios, IRR) with $95 \%$ Cls of obstructive lung diseases as underlying (OLD-u) or underlying or contributing (OLD-uc) cause of death 1951-2007, by cumulative exposure groups, among Norwegian long-term silicon carbide industry workers employed 1913-1920

\begin{tabular}{|c|c|c|c|c|c|c|c|c|c|c|c|c|c|c|c|}
\hline & & \multirow{2}{*}{\multicolumn{2}{|c|}{$\begin{array}{l}\text { All employees } \\
(\mathrm{N}=1687) \\
\text { OLD-u (45 cases) }\end{array}$}} & \multicolumn{6}{|c|}{ All ever-smokers $(\mathrm{N}=1162)$} & \multirow{2}{*}{\multicolumn{3}{|c|}{$\begin{array}{l}<15 \text { years employment, } \\
\text { ever-smokers }(\mathrm{N}=1115) \\
\text { OLD-uc ( } 32 \text { cases) }\end{array}$}} & \multirow{2}{*}{\multicolumn{3}{|c|}{$\begin{array}{l}\geq 15 \text { years employment, } \\
\text { ever-smokers }(N=599) \\
\text { OLD-uc ( } 23 \text { cases) }\end{array}$}} \\
\hline & & & & \multicolumn{3}{|c|}{ OLD-u (38 cases) } & \multicolumn{3}{|c|}{ OLD-uc (55 cases) } & & & & & & \\
\hline & & SMR & $95 \% \mathrm{Cl}$ & IRR* $^{*}$ & $95 \% \mathrm{Cl}$ & $P_{\text {trend }}$ & IRR* & $95 \% \mathrm{Cl}$ & $\mathbf{P}_{\text {trend }}$ & IRR* $^{*}$ & $95 \% \mathrm{Cl}$ & $\mathbf{P}_{\text {trend }}$ & IRR* & $95 \% \mathrm{Cl}$ & $\mathbf{P}_{\text {trend }}$ \\
\hline \multicolumn{16}{|l|}{ Total dust } \\
\hline Low & $0-28.6 \mathrm{mg} \times$ years $/ \mathrm{m}^{3}$ & 1.4 & 0.7 to 2.8 & 1.0 & - & - & 1.0 & - & - & 1.0 & - & - & 1.0 & - & - \\
\hline Medium & $28.7-86.1 \mathrm{mg} \times$ years $/ \mathrm{m}^{3}$ & 2.6 & 1.6 to 4.2 & 2.8 & 0.9 to 8.5 & - & 1.7 & 0.7 to 4.1 & - & 1.3 & 0.5 to 3.4 & - & 2.2 & 0.2 to 24.5 & - \\
\hline High & $\geq 86.2 \mathrm{mg} \times$ years $/ \mathrm{m}^{3}$ & 1.9 & 1.2 to 2.9 & 2.5 & 0.8 to 7.4 & $\mathrm{p}=0.2$ & 2.4 & 1.0 to 5.5 & $p=0.03$ & 2.3 & 0.8 to 6.4 & $p=0.1$ & 5.4 & 0.7 to 40.1 & $p=0.05$ \\
\hline \multicolumn{16}{|c|}{ Respirable dust } \\
\hline Low & $0-3.7 \mathrm{mg} \times$ years $/ \mathrm{m}^{3}$ & 1.5 & 0.8 to 2.8 & 1.0 & - & - & 1.0 & - & - & 1.0 & - & - & 1.0 & - & - \\
\hline Medium & $3.8-9.9 \mathrm{mg} \times$ years $/ \mathrm{m}^{3}$ & 2.7 & 1.7 to 4.3 & 2.0 & 0.8 to 5.1 & - & 1.2 & 0.6 to 2.7 & - & 1.0 & 0.4 to 2.5 & - & 1.2 & 0.2 to 8.7 & - \\
\hline High & $\geq 10 \mathrm{mg} \times$ years $/ \mathrm{m}^{3}$ & 1.8 & 1.1 to 2.9 & 1.5 & 0.6 to 3.9 & $\mathrm{p}=0.5$ & 1.6 & 0.8 to 3.3 & $\mathrm{p}=0.2$ & 1.7 & 0.7 to 4.6 & $\mathrm{p}=0.3$ & 2.4 & 0.6 to 10.2 & $\mathrm{p}=0.2$ \\
\hline \multicolumn{16}{|l|}{ Fibre } \\
\hline Low & $0-0.5$ fibres $\times$ years $/ \mathrm{cm}^{3}$ & 1.9 & 1.1 to 3.4 & 1.0 & - & - & 1.0 & - & - & 1.0 & - & - & 1.0 & - & - \\
\hline Medium & $0.6-2.0$ fibres $\times$ years $/ \mathrm{cm}^{3}$ & 1.9 & 1.1 to 3.3 & 0.9 & 0.4 to 2.3 & - & 0.7 & 0.3 to 1.5 & - & 0.6 & 0.2 to 1.3 & - & 1.8 & 0.3 to 9.8 & - \\
\hline High & $\geq 2.1$ fibres $\times$ years $/ \mathrm{cm}^{3}$ & 2.1 & 1.3 to 3.2 & 1.4 & 0.6 to 3.1 & $\mathrm{p}=0.4$ & 1.2 & 0.6 to 2.2 & $\mathrm{p}=0.5$ & 1.2 & 0.5 to 2.8 & $\mathrm{p}=0.8$ & 2.6 & 0.6 to 11.4 & $\mathrm{p}=0.2$ \\
\hline \multicolumn{16}{|c|}{ Silicon carbide dust } \\
\hline Low & $0-0.7 \mathrm{mg} \times$ years $/ \mathrm{m}^{3}$ & 0.9 & 0.4 to 2.0 & 1.0 & - & - & 1.0 & - & - & 1.0 & - & - & 1.0 & & - \\
\hline Medium & $0.8-2.6 \mathrm{mg} \times$ years $/ \mathrm{m}^{3}$ & 2.8 & 1.7 to 4.4 & 3.1 & 1.0 to 9.3 & - & 1.9 & 0.8 to 4.4 & - & 1.7 & 0.6 to 4.4 & - & 2.4 & 0.5 to 12.1 & - \\
\hline High & $\geq 2.7 \mathrm{mg} \times$ years $/ \mathrm{m}^{3}$ & 2.2 & 1.4 to 3.4 & 3.4 & 1.2 to 10.2 & $p=0.03$ & 2.5 & 1.1 to 5.5 & $p=0.02$ & 2.9 & 1.1 to 7.6 & $p=0.03$ & 2.9 & 0.7 to 12.8 & $\mathrm{p}=0.2$ \\
\hline \multicolumn{16}{|l|}{ Quartz } \\
\hline Low & $0-13.7 \mu \mathrm{g} \times$ years $/ \mathrm{m}^{3}$ & 2.0 & 1.2 to 3.5 & 1.0 & - & - & 1.0 & - & - & 1.0 & - & - & 1.0 & - & - \\
\hline Medium & $13.8-50.3 \mu \mathrm{g} \times$ years $/ \mathrm{m}^{3}$ & 1.7 & 0.9 to 3.1 & 0.7 & 0.3 to 1.8 & - & 0.9 & 0.4 to 2.0 & - & 0.8 & 0.3 to 1.9 & - & 1.9 & 0.2 to 17.0 & - \\
\hline High & $\geq 50.4 \mu \mathrm{g} \times$ years $/ \mathrm{m}^{3}$ & 2.1 & 1.4 to 3.3 & 1.3 & 0.6 to 2.8 & $p=0.4$ & 1.5 & 0.8 to 3.0 & $p=0.2$ & 1.4 & 0.6 to 3.2 & $p=0.4$ & 4.3 & 0.6 to 32.0 & $\mathrm{p}=0.06$ \\
\hline \multicolumn{16}{|l|}{ Cristobalite } \\
\hline Low & $0-10.3 \mu \mathrm{g} \times$ years $/ \mathrm{m}^{3}$ & 1.7 & 0.9 to 3.0 & 1.0 & - & - & 1.0 & & - & 1.0 & & - & 1.0 & - & - \\
\hline Medium & $10.4-47.6 \mu \mathrm{g} \times$ years $/ \mathrm{m}^{3}$ & 1.8 & 1.0 to 3.0 & 0.7 & 0.3 to 1.9 & - & 0.6 & 0.3 to 1.4 & - & 0.7 & 0.3 to 1.7 & - & 0.9 & 0.2 to 4.8 & - \\
\hline High & $\geq 47.7 \mu \mathrm{g} \times$ years $/ \mathrm{m}^{3}$ & 2.4 & 1.6 to 3.7 & 1.6 & 0.7 to 3.4 & $\mathrm{p}=0.2$ & 1.7 & 0.9 to 3.2 & $p=0.05$ & 1.4 & 0.6 to 3.1 & $\mathrm{p}=0.4$ & 3.2 & 0.7 to 14.0 & $p=0.02$ \\
\hline
\end{tabular}

${ }^{*}$ Adjusted for age $(</ \geq 75$ years) and period of diagnosis $(</ \geq 1$ January 1990$)$.

respirable fraction (quartz, cristobalite and $\mathrm{SiC}$ dust, and fibres) were estimated and used in analyses of exposure related mortality from OLD. However, other exposures in the $\mathrm{SiC}$ industry that could be important in this connection, for example, sulphur dioxide and carbonaceous materials such as coke, graphite and polycyclic aromatic hydrocarbons, were not measured in sufficient detail to be included in the JEM. These exposure factors are mainly present in the furnace department. In the processing department, $\mathrm{SiC}$ is the most abundant exposure factor and represents $60-80 \%$ of the respirable dust. In the furnace department, $\mathrm{SiC}$ represents $20-40 \%$ of the respirable dust. $^{10}$

Table 4 Poisson regression analyses (incidence rate ratios, IRR) with 95\% Cls, likelihood ratio test (LR-test) and Pearson's correlation coefficient

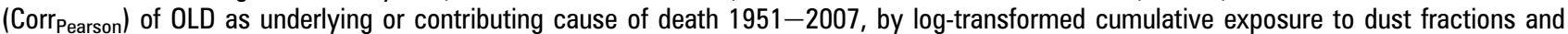
employment duration, among 1162 ever-smoking Norwegian long-term silicon carbide (SiC) industry workers employed 1913-2003

\begin{tabular}{|c|c|c|c|c|c|c|c|c|c|c|c|c|}
\hline & \multicolumn{4}{|c|}{$\begin{array}{l}\text { All }(N=1162), 55 \text { cases, } 30474 \\
\text { person-years }\end{array}$} & \multicolumn{4}{|c|}{$\begin{array}{l}<15 \text { years of employment duration } \\
(\mathrm{N}=1115), 32 \text { cases, } 18906 \text { person-years }\end{array}$} & \multicolumn{4}{|c|}{$\begin{array}{l}\geq 15 \text { years of total employment duration } \\
(N=599), 23 \text { cases, } 11567 \text { person-years }\end{array}$} \\
\hline & IRR* $^{*}$ & $95 \% \mathrm{Cl}$ & LR-test $†$ & Corr $_{\text {Pearson }}$ & IRR* $^{*}$ & $95 \% \mathrm{Cl}$ & LR-test $\dagger$ & Corr $_{\text {Pearson }}$ & IRR* $^{*}$ & $95 \% \mathrm{CI}$ & LR-test $\dagger$ & Corr $_{\text {Pearson }}$ \\
\hline Fibre & 1.3 & 0.9 to 2.0 & - & - & 1.0 & 0.6 to 1.9 & - & - & 2.4 & 1.1 to 5.4 & - & - \\
\hline Fibre adjusted for quartz & 1.0 & 0.6 to 1.8 & $\mathrm{p}=0.2$ & 0.80 & 0.7 & 0.3 to 1.7 & $\mathrm{p}=0.3$ & 0.76 & 1.6 & 0.5 to 4.5 & $\mathrm{p}=0.2$ & 0.83 \\
\hline Fibre adjusted for cristobalite & 1.0 & 0.6 to 1.7 & $\mathrm{p}=0.1$ & 0.69 & 0.8 & 0.4 to 1.9 & $\mathrm{p}=0.5$ & 0.69 & 1.3 & 0.5 to 3.3 & $p=0.03$ & 0.67 \\
\hline Fibre adjusted for SiC & 1.0 & 0.6 to 1.6 & $p=0.05$ & 0.66 & 0.9 & 0.4 to 1.7 & $p=0.03$ & 0.54 & 2.3 & 0.9 to 5.9 & $\mathrm{p}=0.9$ & 0.75 \\
\hline SiC particles & 1.6 & 1.1 to 2.5 & - & - & 2.0 & 1.0 to 3.7 & - & - & 1.6 & 0.9 to 3.2 & - & - \\
\hline SiC adjusted for quartz & 1.5 & 1.0 to 2.4 & $\mathrm{p}=0.3$ & 0.55 & 2.0 & 1.0 to 3.7 & $\mathrm{p}=0.4$ & 0.44 & 1.3 & 0.6 to 2.7 & $p=0.03$ & 0.64 \\
\hline SiC adjusted for cristobalite & 1.5 & 0.9 to 2.3 & $\mathrm{p}=0.2$ & 0.54 & 2.0 & 1.0 to 3.9 & $\mathrm{p}=0.9$ & 0.49 & 1.0 & 0.5 to 2.2 & $\mathrm{p}=0.005$ & 0.57 \\
\hline SiC adjusted for fibre & 1.6 & 1.0 to 2.7 & $\mathrm{p}=0.9$ & 0.66 & 2.0 & 1.1 to 3.8 & $\mathrm{p}=0.7$ & 0.54 & 1.1 & 0.4 to 2.7 & $p=0.06$ & 0.75 \\
\hline Quartz & 1.5 & 1.0 to 2.3 & - & - & 1.3 & 0.7 to 2.5 & - & - & 2.7 & 1.2 to 5.9 & - & - \\
\hline Quartz adjusted for cristobalite & 1.2 & 0.6 to 2.2 & $\mathrm{p}=0.2$ & 0.71 & 1.3 & 0.6 to 2.9 & $\mathrm{p}=0.9$ & 0.68 & 1.4 & 0.5 to 4.4 & $p=0.04$ & 0.72 \\
\hline Quartz adjusted for fibre & 1.5 & 0.8 to 2.9 & $\mathrm{p}=1.0$ & 0.80 & 1.6 & 0.7 to 3.8 & $\mathrm{p}=0.5$ & 0.76 & 2.0 & 0.7 to 5.9 & $p=0.4$ & 0.83 \\
\hline Quartz adjusted for SiC & 1.3 & 0.8 to 2.1 & $p=0.06$ & 0.55 & 1.3 & 0.6 to 2.8 & $p=0.03$ & 0.44 & 2.6 & 1.1 to 5.9 & $\mathrm{p}=0.5$ & 0.64 \\
\hline Cristobalite & 1.4 & 1.0 to 2.0 & - & - & 1.1 & 0.8 to 1.7 & - & - & 2.5 & 1.4 to 4.3 & - & - \\
\hline Cristobalite adjusted for quartz & 1.3 & 0.8 to 2.0 & $\mathrm{p}=0.6$ & 0.71 & 1.0 & 0.6 to 1.7 & $\mathrm{p}=0.5$ & 0.68 & 2.1 & 1.0 to 4.4 & $\mathrm{p}=0.6$ & 0.72 \\
\hline Cristobalite adjusted for fibre & 1.4 & 0.9 to 2.2 & $\mathrm{p}=1.0$ & 0.69 & 1.2 & 0.7 to 2.1 & $\mathrm{p}=0.7$ & 0.69 & 2.2 & 1.1 to 4.5 & $\mathrm{p}=0.6$ & 0.67 \\
\hline Cristobalite adjusted for SiC & 1.2 & 0.9 to 1.8 & $\mathrm{p}=0.1$ & 0.54 & 1.0 & 0.6 to 1.5 & $p=0.04$ & 0.49 & 2.4 & 1.3 to 4.5 & $\mathrm{p}=0.9$ & 0.57 \\
\hline
\end{tabular}

*Adjusted for age $(</ \geq 75$ years) and period of diagnosis $(</ \geq 1$ January 1990).

†LR-test: likelihood ratio test comparing the model containing one exposure factor with a model containing two exposure factors. 
A priori we applied categories of cumulative exposure by equal number of person-years in each group in our analyses. Post hoc, we have tested categories based on equal numbers of OLD cases in each group. The results are quite similar. Another alternative could be to choose cut-points from the cumulative exposure data, based on presumed low, medium and high risk levels. This was not attempted because we had no such presuppositions, and arbitrarily chosen cut-points would give a greater risk of collapse in the analyses because of uneven distribution of the cases.

Development of the JEM used in the present study was based on dust measurements performed since 1967. The exposure estimates for the period before 1967 were based on information about changes in processes and working hours. Hence, the exposure estimates before 1967 were less reliable than the later estimates. However, the exposure estimates were developed independently of the outcomes from the epidemiological study, giving a non-differential misclassification of exposure, which in most cases will give a bias towards the null. ${ }^{14}$

The cohort used in this study has a cross-sectional start in 1942, with start of follow-up in 1951. Thus, the oldest part of the cohort consists of survivors from the period 1913-1951. As exposure intensities were higher in earlier times, one would expect that work related death was also higher in earlier times. In the Norwegian $\mathrm{SiC}$ industry, many silicosis cases were reported in the 1940s, and there is reason to believe that quite a few workers died from work related lung diseases, primarily silicosis, with and without tuberculosis, in the 10-20years before $1950 .^{1}{ }^{2}$ This would result in a healthy survivor population at start of follow-up in 1951, probably giving an underestimation of work related mortality in the present study. The fact that we observe only two cases of OLD-u (three cases of OLDuc) in the period 1951-1969 (table 2) may also point in the direction of a healthy survivor cohort. On the other hand, we do not have any reason to believe that the left-censoring caused an overestimation of the risk. We have also performed analyses with an inception subcohort starting in 1951, and the overall trends are the same as presented in table 3. Further subdivision of the cohort, at 15 years of employment, results in small groups, with fragile and unreliable results (results not shown).

One interesting finding in this study was the contrast between exposure-response associations among workers with less than and more than 15 years of employment in the $\mathrm{SiC}$ industry. Occupational exposure to minerals and fibres can elicit symptom-producing responses in the airways, often clinically interpreted as OLD ${ }^{15}$ through different mechanisms, depending on the characteristics of the dust, for example, chemical composition, particle size and surface properties. ${ }^{16} 17$ Other risk factors for OLD are familiar disposition, smoking and earlier infections. ${ }^{18}$ Subjects with a higher risk of OLD from a combination of causes ${ }^{1920}$ may have an increased risk of early airway symptoms in a dusty work environment. This could, eventually, lead to selection out of exposed work. ${ }^{21}$ The toxicity of $\mathrm{SiC}$ dust has previously been studied in in vitro and in vivo experiments, and it has been classified as 'inert'. ${ }^{22} 23$ Our finding of an association between $\mathrm{SiC}$ and OLD mortality among workers who left the industry before 15 years of employment, and also the close correlation between $\mathrm{SiC}$ and total dust may, however, be consistent with $\mathrm{SiC}$ being part of a nuisance dust load which may induce OLD symptoms among disposed individuals. The limitation of this association to workers with less than 15 years of employment may be suggestive of a healthy survivor effect. ${ }^{12}$

Crystalline silica, on the other hand, is present in lower concentrations, and represents only a minor fraction of the total dust exposure in the $\mathrm{SiC}$ industry. Crystalline silica will, however, accumulate in the lung, ${ }^{24}$ and may induce lung injury, with symptoms associated with OLD that do not appear until many years after first exposure. ${ }^{25}$ This could explain our findings of exposure-response associations with cristobalite among the workers with more than 15 years of employment. Quartz seemed to be a less important exposure factor than cristobalite. Previous studies have indicated that cristobalite is a more potent fibrogen than quartz. ${ }^{26} 27$ The results of our study suggest that cristobalite is also more potent than quartz when OLD is the outcome. When we summed the exposure to cristobalite and quartz, generating a log-transformed cumulative crystalline silica exposure variable, we found a stronger exposure-response association (IRR 3.1, 95\% CI 1.5 to 6.3) than with cristobalite alone, indicating an additive effect of quartz and cristobalite.

As smoking is the most important cause of OLD, adjustment for smoking is necessary in studies of other aetiological factors. Among the long-term workers in the present cohort, 69\% were ever-smokers, and only seven of the cases (11\%) were among never-smokers or people with unknown smoking status. The small numbers of cases in the latter two groups caused a collapse in the multivariate analyses with adjustment for smoking, and we therefore chose to perform these analyses in the subcohort of ever-smokers. Our findings may still be biased by a possible correlation between smoking and degree of dust exposure, or even an interaction between these factors, but we do not have the information needed to address this question.

Mortality from pneumoconiosis, which is exclusively a work related disease, was increased to a high level (SMR 15, 95\% CI 7.0 to 31 ). It would have been of interest to study exposureresponse associations with pneumoconiosis using the JEM, but the numbers were too small to obtain meaningful results (seven cases). All those who died from pneumoconiosis had a diagnosis of silicosis, had worked in the SiC industry for more than 20 years before 1975, and had first employment before 1950. They had been exposed in different departments, including the furnace, processing and maintenance sections.

The mortality from pneumonia was significantly increased with 38 deaths and an SMR of 1.4. A review of the contributing causes of death gave no indication of work relatedness-only one person had COPD as contributing cause of death, and many had dementia and prostate cancer, associated with high age (mean age at death: 84 years). We do not believe that this increased incidence of pneumonia was related to occupational exposure in the $\mathrm{SiC}$ industry.

\section{CONCLUSIONS}

We have in the present study performed analyses on associations between OLD and cumulative exposure to certain dust species among workers in the SiC industry. Mortality from OLD was increased compared to the general population, and significantly increased risks were seen at the highest level of cumulative exposure to all investigated exposure factors. Exposure-response relationships were found with $\mathrm{SiC}$ and crystalline silica. However, other unmeasured components in the furnace hall, such as carbonaceous dust and sulphur dioxide, may also have contributed to the observed excess of OLD. Different exposure factors may contribute to OLD development among $\mathrm{SiC}$ workers in different time-windows, possibly through different mechanisms.

Acknowledgements The authors thank Pål Romundstad and Berit Bakke for commenting on the manuscript draft.

Funding The project has been financed with the aid of EXTRA funds from the Norwegian Foundation for Health and Rehabilitation, and with support from the Ministry of Labour and Social Inclusion. 


\section{Competing interests None.}

Ethics approval This study was conducted with the approval of the Regional Committee for Medical Research Ethics South-East Norway, Oslo University, Norway.

Provenance and peer review Not commissioned; externally peer reviewed.

\section{REFERENCES}

1. Winslow C-EA, Greenburg L, Greenberg D. The dust hazard in the abrasive industry. Public Health Rep 1919;530:1171-87.

2. Bruusgaard A. Pneumoconiosis in silicon carbide workers. Proceedings of the 9th International Congress on Industrial Medicine. London, Bristol: Wright, 1948:676-80.

3. Infante-Rivard C, Dufresne A, Armstrong B, et al. Cohort study of silicon carbide production workers. Am J Epidemiol 1994;140:1009-15.

4. Romundstad $\mathbf{P}$, Andersen A, Haldorsen T. Non-malignant mortality among Norwegian silicon carbide smelter workers. Occup Environ Med 2002;59:345-7.

5. Liethschmidt K. Silicon carbide. Ullmann's Encyclopedia of Industrial Chemistry. 5th edn. Weinheim, Germany: VCH Verlagsgesellshaft mbH, 1993:749-58.

6. Bye E, Eduard W, Gjønnes J, et al. Occurrence of airborne silicon carbide fibers during industrial production of silicon carbide. Scand J Work Environ Health 1985:11:111-15.

7. Bugge MD, Kjuus $\mathrm{H}$, Martinsen Jl, et al. Cancer incidence among short- and longterm workers in the Norwegian silicon carbide industry. Scand J Work Environ Health 2010;36:71-9.

8. Holten K, Tofte T. [Obstructive lung disease in a department of respiratory medicine 1970-88]. Tidsskr Nor Laegeforen 1997:117:1585-9.

9. Frostad A, Soyseth V, Haldorsen $T$, et al. Respiratory symptoms and 30 year mortality from obstructive lung disease and pneumonia. Thorax 2006;61:951-6.

10. Foreland S, Bye E, Bakke B, et al. Exposure to fibres, crystalline silica, silicon carbide and sulphur dioxide in the norwegian silicon carbide industry. Ann Occup Hyg 2008;52:317-36.

11. Seixas NS, Robins TG, Moulton LH. The use of geometric and arithmetic mean exposures in occupational epidemiology. Am J Ind Med 1988;14:465-77.

12. Attfield MD, Kuempel ED. Mortality among U.S. underground coal miners: a 23-year follow-up. Am J Ind Med 2008;51:231-45.
13. Kirkwood BR, Sterne JAC. Essential Medical Statistics. 2nd edn. Malden, Massachusetts: Blackwell Science, 2006:249-62.

14. Checkoway H, Pearce N, Kriebel D. Research Methods in Occupational Epidemiology. 2nd edn. New York: Oxford University Press, 2004:107-9.

15. Becklake MR. Chronic airflow limitation: its relationship to work in dusty occupations. Chest 1985;88:608-17.

16. Bruch J, Rehn S, Rehn B, et al. Variation of biological responses to different respirable quartz flours determined by a vector model. Int J Hyg Environ Health 2004:207:203-16.

17. Governa $\mathbf{M}$, Valentino $\mathbf{M}$, Amati $\mathbf{M}$, et al. Reactive oxygen species measured from suspensions of polymorphonuclear leukocytes after the addition of silicon carbide particles. Altern Lab Anim 1996:24:553-6.

18. Roisin RR, Anzueto A, Bourbeau J, et al. Global Strategy for the Diagnosis, Management and Prevention of COPD, Global Initiative for Chronic Obstructive Lung Disease (GOLD). 2010:16-21. Available from: http://www.goldcopd.org (Accessed 21 February 2011).

19. Pavord ID, Birring SS, Berry M, et al. Multiple inflammatory hits and the pathogenesis of severe airway disease. Eur Respir J 2006;27:884-8.

20. Hendrick DJ. Occupation and chronic obstructive pulmonary disease (COPD) Thorax 1996:51:947-55.

21. Søyseth V, Johnsen HL, Kongerud J. Prediction of dropout from respiratory symptoms and airflow limitation in a longitudinal respiratory study. Scand J Work Environ Health 2008:34:224-9.

22. Bruch J, Rehn B, Song $H$, et al. Toxicological investigations on silicon carbide. 1. Inhalation studies. Br J Ind Med 1993;50:797-806.

23. Bruch J, Rehn B, Song W, et al. Toxicological investigations on silicon carbide. 2. In vitro cell tests and long term injection tests. Br J Ind Med 1993;50:807-13.

24. International Agency for Research on Cancer. Silica, Some Silicates, Coal Dust and Para-Amid Fibrils. IARC Monographs on the Evaluation of Carcinogenic Risks to Humans. Lyon: IARC, 1997:176-9.

25. Hnizdo E, Vallyathan V. Chronic obstructive pulmonary disease due to occupational exposure to silica dust: a review of epidemiological and pathological evidence. Occup Environ Med 2003;60:237-43.

26. King EJ, Mohanty GP, Harrison CV, et al. The action of different forms of pure silica on the lungs of rats. Br J Ind Med 1953;10:9-17.

27. Hemenway DR, Absher MP, Trombley $\mathrm{L}$, et al. Comparative clearance of quartz and cristobalite from the lung. Am Ind Hyg Assoc J 1990;51:363-9.

\section{OEM online}

Visit OEM online for free editor's choice articles, top ten articles, online archive, blogs and online first articles. Keep informed and up to date by registering for electronic table of contents at oem. bmj.com. 